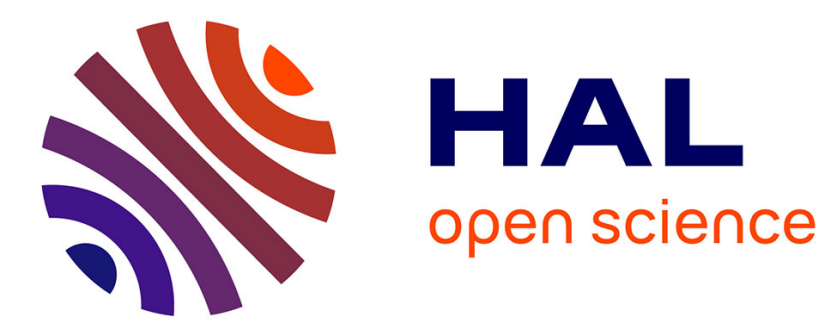

\title{
Mesures de vies moyennes par effet Doppler
}

\author{
A. Gallmann, F. Haas, B. Heusch, M. Toulemonde
}

\section{To cite this version:}

A. Gallmann, F. Haas, B. Heusch, M. Toulemonde. Mesures de vies moyennes par effet Doppler. Revue de Physique Appliquée, 1969, 4 (2), pp.216-217. 10.1051/rphysap:0196900402021601 . jpa-00243227

\section{HAL Id: jpa-00243227 https://hal.science/jpa-00243227}

Submitted on 1 Jan 1969

HAL is a multi-disciplinary open access archive for the deposit and dissemination of scientific research documents, whether they are published or not. The documents may come from teaching and research institutions in France or abroad, or from public or private research centers.
L'archive ouverte pluridisciplinaire HAL, est destinée au dépôt et à la diffusion de documents scientifiques de niveau recherche, publiés ou non, émanant des établissements d'enseignement et de recherche français ou étrangers, des laboratoires publics ou privés. 


\title{
MESURES DE VIES MOYENNES PAR EFFET DOPPLER
}

\author{
A. GALLMANN, F. HAAS, B. HEUSCH et M. TOULEMONDE, \\ Gentre de Recherches Nucléaires de Strasbourg-Cronenbourg.
}

Résumé. - Des vies moyennes de niveaux nucléaires ont été déterminées par la méthode de l'effet Doppler. Les rayonnements gamma de désexcitation ont été détectés dans des compteurs $\mathrm{Ge}(\mathrm{Li})$. Des vies moyennes ont été obtenues pour les niveaux $0,953 \mathrm{MeV}$ de ${ }^{12} \mathrm{~B}$ et $6,44 \mathrm{MeV}$ de ${ }^{14} \mathrm{~N}$.

Abstract. - Lifetimes of nuclear states have been obtained by the Doppler shift attenuation method. The $\gamma$-rays were detected in lithium-drifted germanium counters. Lifetimes of the ${ }^{12} \mathrm{~B} 0.953 \mathrm{MeV}$ and the ${ }^{14} \mathrm{~N} 6.44 \mathrm{MeV}$ levels are reported.

La possibilité d'utiliser des compteurs $\mathrm{Ge}(\mathrm{Li})$ de très bonne résolution permet actuellement la mesure de vies moyennes nucléaires comprises entre $10^{-12}$ et $5 \times 10^{-15} \mathrm{~s}$. La mesure des déplacements en énergie des raies $\gamma$ est bien plus précise qu'avec les détecteurs $\mathrm{NaI}$ utilisés précédemment. D'autre part, la résolution des $\mathrm{Ge}(\mathrm{Li})$ est généralement de l'ordre de grandeur ou inférieure aux déplacements Doppler; il est alors possible d'étudier les distributions d'énergies des rayonnements gamma observés.

Les rayonnements gamma des réactions

$$
{ }^{11} \mathrm{~B}(\mathrm{~d}, \mathrm{p}){ }^{12} \mathrm{~B}(1) \quad \text { et } \quad{ }^{12} \mathrm{C}\left({ }^{3} \mathrm{He}, \mathrm{p}\right){ }^{14} \mathrm{~N}(2)
$$

à des énergies incidentes de 2,1 et $4,0 \mathrm{MeV}$ respectivement, ont été détectés dans un compteur $\mathrm{Ge}(\mathrm{Li})$ de $22 \mathrm{~cm}^{3}$. L'exploitation des spectres gamma enregistrés à $\theta \gamma=7^{\circ}, 90^{\circ}$ et $173^{\circ}$, se faisait de la façon suivante : les déplacements en énergie des rayonnements gamma dus à l'effet Doppler ont été obtenus, après soustraction d'un bruit de fond exponentiel sous les pics étudiés, par la détermination des centres de gravité des pics aux trois angles. Les déplacements des raies $\gamma$ ont été analysés en tenant compte de la perte d'énergie des ions de recul dans les milieux ralentisseurs, l'or dans le cas de la réaction (1), le 


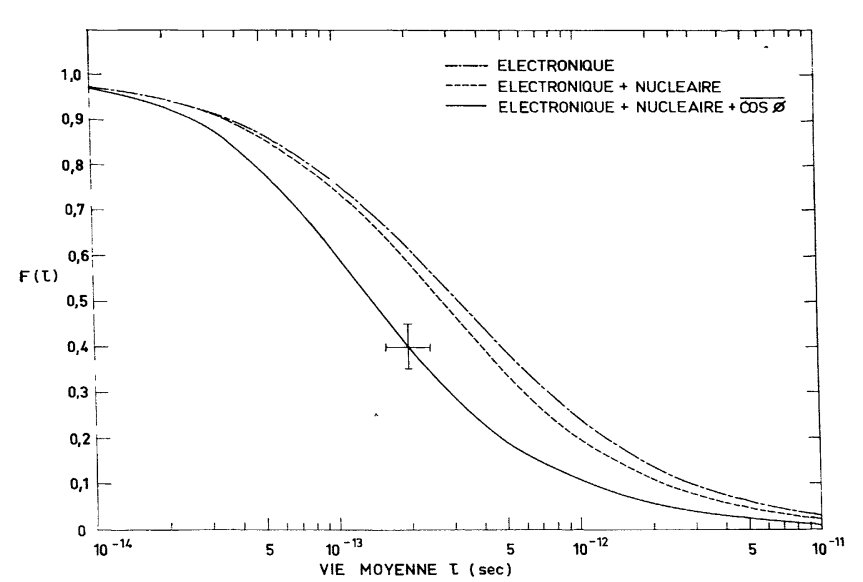

Fig. 1. - Niveau $0,953 \mathrm{MeV}$ de ${ }^{12} \mathrm{~B}$ : variation du facteur d'atténuation $F(\tau)$ en fonction de la vie moyenne $\tau$.

carbone pour la réaction (2). La méthode d'analyse adoptée est celle décrite par Blaugrund [1]. La figure 1 montre le résultat obtenu pour le niveau de $0,953 \mathrm{MeV}$ de ${ }^{12} \mathrm{~B}$. Le facteur $F(\tau)$ représente le rapport des déplacements observés pour des ions de ${ }^{12} \mathrm{~B}$ reculant respectivement dans l'or et le vide (dans ce dernier cas, l'ion recule avec sa vitesse initiale).

Dans certaines conditions idéales de ralentissement et de déplacement, il est également possible d'étudier la distribution d'énergie des rayonnements gamma. Un programme d'analyse de la forme des pics a été conçu, tenant compte de la résolution du détecteur, du milieu ralentisseur, de la vitesse initiale des noyaux de recul, ainsi que de leur distribution angulaire. Cette méthode d'analyse décrite par le groupe de Brookhaven [2] nous permet de déterminer des vies moyennes précises et, d'autre part, nous renseigne sur le mécanisme de perte d'énergie et de diffusion des ions dans la matière. Un exemple d'analyse de la forme d'une raie est représenté sur la figure 2 pour la transition $(6,44 \rightarrow 0)$ dans le noyau ${ }^{14} \mathrm{~N}$. Les formes de la raie ont été cal-

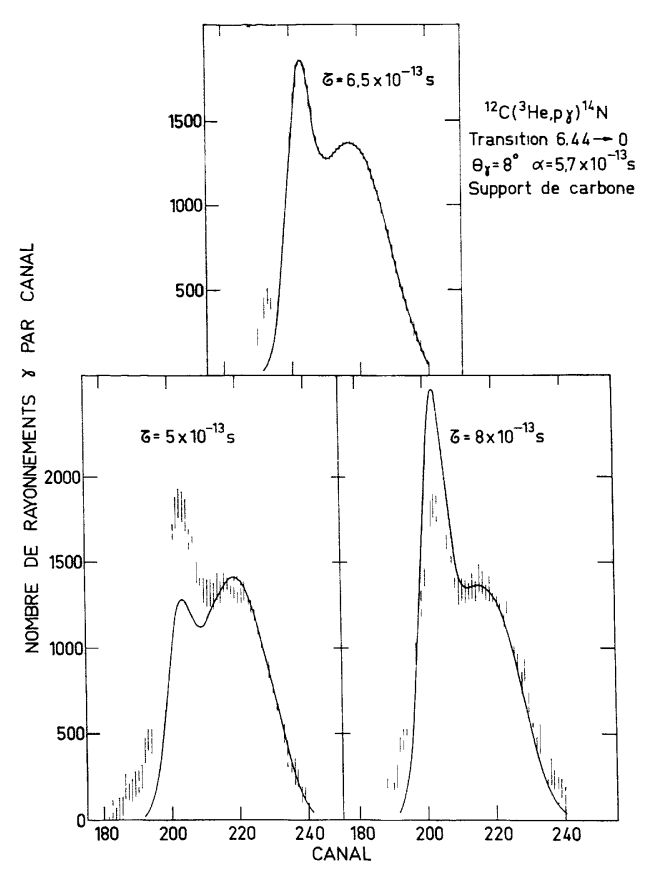

FIG. 2. - Niveau 6,44 $\mathrm{MeV}$ de ${ }^{14} \mathrm{~N}$ : formes de la raie $\gamma$ de la transition $(6,440 \rightarrow 0)$ du noyau ${ }^{14} \mathrm{~N}$ pour des temps de vie $\tau=5,0 \times 10^{-13} \mathrm{~s}, 6,5 \times 10^{-13} \mathrm{~s}$ et $8,0 \times 10^{-13} \mathrm{~s}$ comparées à la forme expérimentale.

culées pour des temps de vie compris entre $10^{-14} \mathrm{~s}$ et $10^{-11} \mathrm{~s}$. Sur la figure 2 , qui est une illustration de cette étude, trois formes sont représentées, ainsi que la forme expérimentale.

Les temps de vie obtenus pour les deux niveaux considérés sont les suivants :

1) niveau $0,953 \mathrm{MeV}$ de ${ }^{12} \mathrm{~B}$ :

$$
\tau=2,0 \pm 0,4 \times 10^{-13} \mathrm{~s}
$$

2) niveau $6,440 \mathrm{MeV}$ de ${ }^{14} \mathrm{~N}$ :

$$
\tau=6,5 \pm 0,5 \times 10^{-13} \mathrm{~s} .
$$

\section{BIBLIOGRAPHIE}

[1] Blaugrund (A. E.), Nucl. Phys., 1966, 88, 501.

[2] Warburton (E. K.), Olness (J. W.) et Poleitit

(A. R.), Phys. Rev., 1967, 160, 938. 